\title{
Determination of astrometry and photometry of faint companions in the presence of residual speckle noise
}

\author{
Daniel Burke ${ }^{1, a}$, Nicholas Devaney ${ }^{1}$, and Szymon Gladysz ${ }^{2}$ \\ 1 Applied Optics Group \\ School of Physics \\ National University of Ireland, Galway \\ Ireland \\ 2 European Organisation for Astronomical Research in the Southern Hemisphere, \\ Karl-Schwarzschild-Strasse 2, \\ D-85748 Garching bei Munchen, \\ Germany
}

\begin{abstract}
In this paper we examine approaches to faint companion detection and estimation in multi-spectral images. We will employ the Hotelling observer which is the optimal linear algorithm for signal detection. We have shown how to use this observer to estimate faint object position and brightness in the presence of residual speckle which usually limit astrometric and photometric techniques. These speckles can be reduced by differential imaging techniques such as Angular Differential Imaging and Spectral Differential Imaging. Here we present results based on simulations of adaptive optics corrected images from an ELT which contain quasi-static speckle noise. The simulation includes Angular Differential Imaging to reduce the residual speckle and subsequent multi-wavelenght processing. We examine the feasibility of this approach on simulated ELT observations of faint companions.
\end{abstract}

\section{Introduction}

Differential astrometry and photometry of faint sub-stellar companions is difficult due to the high contrast ratio between the parent star and the companion, typically of the order of $10^{-4}$ [4]. When adaptive optics is employed the direct imaging of faint companions is limited by residual quasi-static speckles present in the point spread function (PSF) of the parent star [1].

We propose to use a combination of techniques to overcome this limit. Multi-wavelength data can be used to produce an estimate of the point spread function (PSF). This wavelength diversity method is an extension of phase diversity, proposed by Gonsalves [7]. This approach is combined with classical differential imaging techniques to reduce the effects of speckle noise and the final image is processed with a prewhitening matched filter, referred to as the Hotelling observer, to perform the differential photometry and astrometry.

In $\S 2$ the PSF reconstruction routine will be outlined, the Hotelling observer will be introduced and a brief description will be given of how our test data was generated. $\S 3$ will focus on our application of PSF reconstruction and the Hotelling observer to data which has been reduced using Angular Differential Imaging (ADI). The paper concludes in $\$ 3$ with a discussion of the performance of the algorithm and some suggestions for future study.

\footnotetext{
a e-mail: daniel.burke@nuigalway.ie
} the original work is properly cited. 


\section{Proposed Method}

\subsection{The Hotelling observer}

The Hotelling Observer [2] is sometimes referred to as a prewhitening matched filter. In the process of prewhitening, the data is divided by the data covariance matrix with the aim of producing spatially stationary, uncorrelated noise. The data covariance model requires a good PSF estimate; the PSF estimate is used to subtract the signal of the bright star from the image (PSF subtraction), flatten the residuals (prewhitening), and estimate the companion signal via matched fitlering, (see figure 1). The Hotelling observer provides a framework to include spatial and temporal correlation information about the noise, as well as knowledge about the statistics of the random PSF.

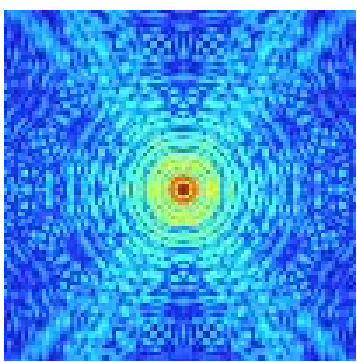

(a) Raw Data

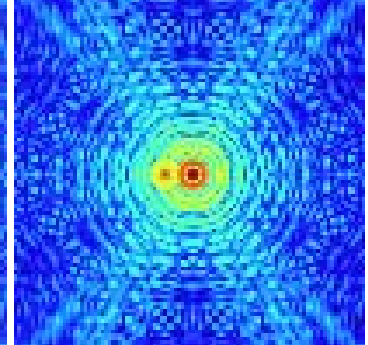

(b) PSF Subtracted Data

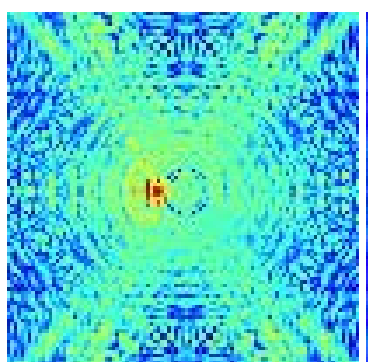

(c) Prewhitened Data

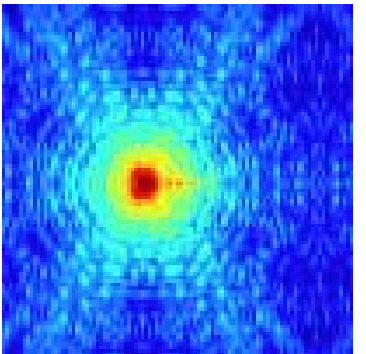

(d) Hotelling test statistic map

Fig. 1: Steps in the application of the Hotelling Observer

The observer calculates a linear discriminant of the form $t(g)=w^{t} g$, where $w$ is called the template, $g$ is the data and $t(g)$ is the test statistic. For each test location the scanning Hotelling observer calculates its test statistic for a set of test intensities. This set of test statistics is at maximum at the location of the companion (see figure 1.d).

\subsection{Observer Comparison}

Artificial binaries $\left(\Delta m_{K}=3.5\right.$ or 4.5 , separation $\left.\theta=0.6^{\prime \prime}\right)$ were simulated by scaling and shifting single-star images from the Lick AO system [6]. For each case 8 positions for the companion were tested in order to minimise the bias from anisotropy in the PSF. The Hotelling Observer was compared to two other widely used algorithms, (figure 2). For details of this comparison see Burke et al. (2009) [3].

The Hotelling algorithm derived the most accurate estimates for the position and intensity of the companion. The minimum error in astrometry achieved using this approach was 0.07 mas, the corresponding error in photometry, at this estimated position, was 0.01 magnitudes.

\subsection{PSF reconstruction using wavelength diversity}

Classical phase-diversity techniques provide an observational method to estimate the phase of the incoming wavefront at the pupil from focal plane intensity measurements. In the usual phase diversity observations two images are recorded; one in focus and a second with a known amount of defocus. Typically the method is based on including a known phase function in the system's image formation process e.g.:

$$
P_{k}(u)=A(u) \exp \left\{i\left[\phi(u)+\theta_{k}(u)\right]\right\} .
$$




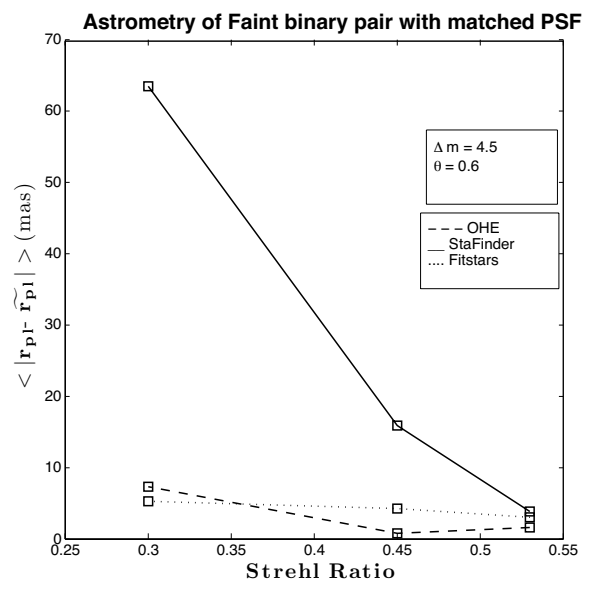

(a) Relative Astrometry

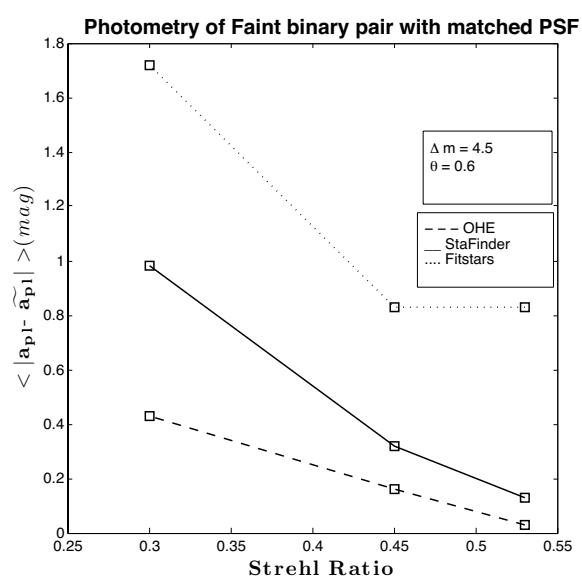

(b) Relative Photometry

Fig. 2: Comparison of the Hotelling Observer (OHE) to two other widely used algorithms.

Where $A(u)$ is the pupil transmission function, $\phi(u)$ is an unknown phase-abberation, $\theta_{k}(u)$ is the known aberration function and $u$ is the spatial frequency. The PSF is the squared modulus of the discrete Fourier Transform of equation (1) and $k$ is the $k$ th diversity channel. Here we study whether multi-wavelength data can be used to retrieve the unknown phase of the wavefront. Instead of adding a known amount of defocus to the unknown phase, the phase-abberation function, $\theta_{k}$, is a factor which scales with wavelength:

$$
P_{k}(u)=A(u) \exp \left\{i\left[\theta_{k} \phi(u)\right]\right\} .
$$

Zernike polynomials are used to expand the phase over a circular pupil:

$$
\phi(u)=\sum_{m=1}^{M} \alpha_{m} \Psi_{m}(u) .
$$

The solution to finding the vector of zernike coefficients which best describes $\phi(u)$ is found by estimating an aberration vector which the minimizes an objective function. The objective function we employed, minimises the least square error between the data and our model, which relies on a Gaussian noise model [7]. The sign of the Zernike polynomials with even radial order is ambiguous when derived from focal plane images. The corresponding PSF is however valid for post-processing.

\subsection{Simulating an ELT: PAOLA}

The end-to-end adaptive optics simulation package PAOLA (Performance of Adaptive Optics for Large/Little Apertures) [8] was used to simulate an Extremely Large Telescope (ELT). PAOLA is not a Monte-Carlo simulation; the algorithm is based on an analytical description of the residual phase spatial power spectrum [5]. For this work a $42 \mathrm{~m}$ primary mirror was modelled with an $12.5 \mathrm{~m}$ central obstruction and four spider arms (figure 3).

\section{Results from Differential Imaging}

Data with a faint companion present was simulated at $1.64 \mu \mathrm{m}$ and $1.8 \mu \mathrm{m}$ and at several different orientations (figure 4). 


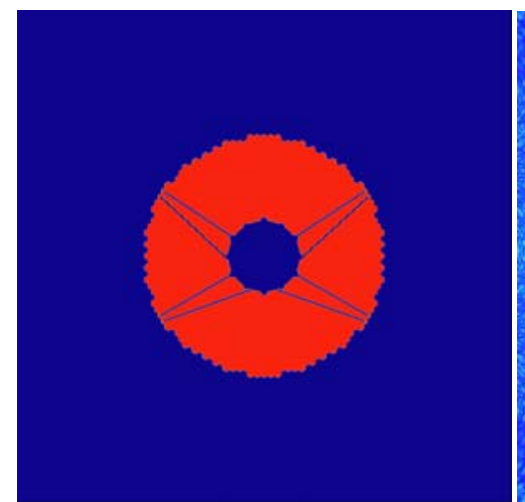

(a) ELT Pupil

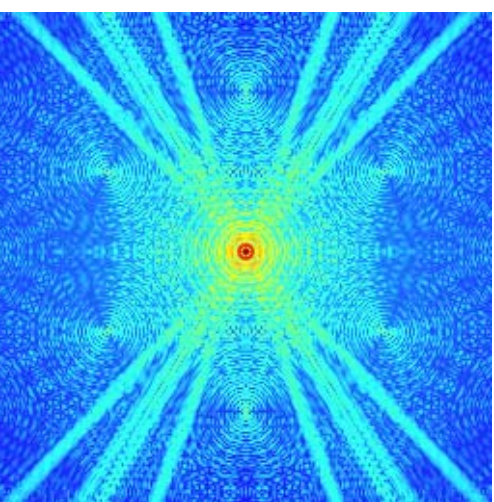

(b) ELT PSF

Fig. 3: Pupil image and PSF of an ELT simulated with the PAOLA package

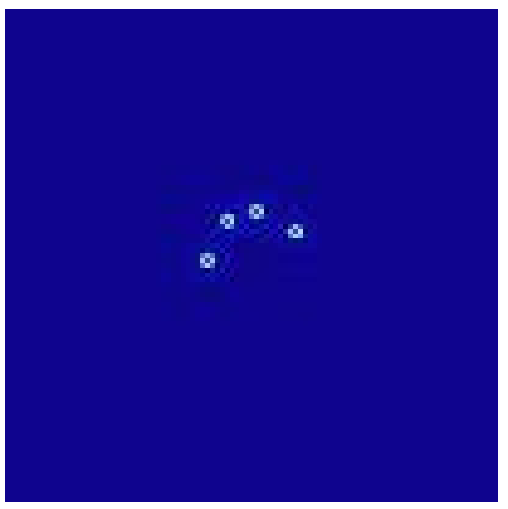

Fig. 4: Angular Differential Imaging Sequence

Table 1: Estimated position and intensity of a faint companion using the Hotelling Observer.

\begin{tabular}{ll}
\hline $\begin{array}{l}\text { Radius of companion } \\
(\operatorname{arcsec})\end{array}$ & $\begin{array}{l}\text { Relative Magnitude } \\
\text { (magnitudes) }\end{array}$ \\
\hline 0.042 (true) & $\Delta m=10$ (true) \\
0.046 (estimated) & $\Delta m=10.4$ (estimated) \\
\hline
\end{tabular}

For each orientation, wavelength diversity was used to estimate the PSF. Angular Differential Imaging was then used to reduce the data at $1.64 \mu \mathrm{m}$, e.g. the image sequence was de-rotated and coadded, see figure 5a. The Hotelling Observer was then used to scan this co-added image and shows a clear maximum at the location of the companion, see figure $5 \mathrm{~b}$, with a corresponding estimate of the intensity of the companion, see table 1.

\section{Summary}

Our combination of differential imaging, wavelength diversity and the Hotelling observer has yielded very accurate differential astrometry and photometry of a faint companion in the presence of quasi- 


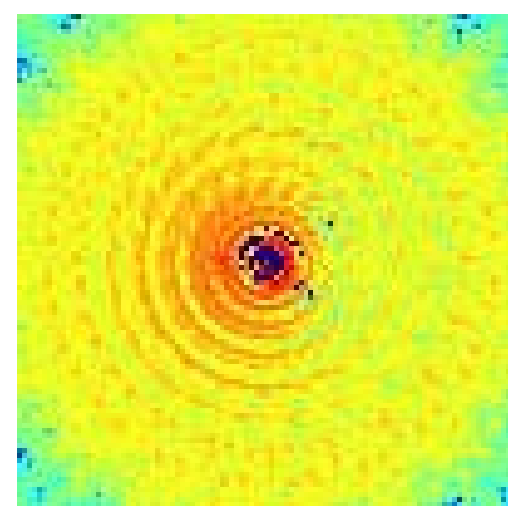

(a) ADI reduced image

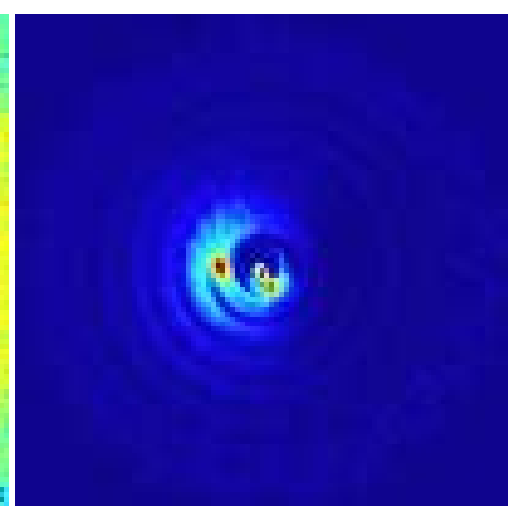

(b) Hotelling test statistic map

Fig. 5: Resulting speckle reduced image and Hotelling test statistic map

static speckle noise. In the future we will investigate the performance of our approach on fainter and closer companions.

Here we assumed that the companion was so faint it did not affect our phase estimates. We are currently investigating methods to include the presence of the companion in our modelling of the PSF from multi-wavelength data. This research was funded by Science Foundation Ireland Grant No. 07/IN.1/1906.

\section{References}

1. Aime and Soummer. The usefulness and limits of coronagraphy in the presence of pinned speckles. Ap.J, 612:85-88, September 2004.

2. H. H. Barrett, K. J. Myers, M. N. Devaney, and J. C. Dainty. "Objective assessment of image quality. IV. Application to adaptive optics". JOSA A, 23(12):3080-3105, 2006.

3. Burke, Daniel and Gladysz, Szymon and Roberts, Lewis and Devaney, Nicholas and Dainty, Chris. An Improved Technique for the Photometry and Astrometry of Faint Companions. Publications of the Astronomical Society of the Pacific, 121(881):767-777, 2009.

4. Adam Burrows. A theoretical look at the direct detection of giant planets outside the solar system. Nature, 433(7023):261-268, 012005.

5. S. Gladysz, E. Ribak, B. F. Castella, L. Jolissaint, P. Martinez, R. Soummer, E. A. Carpentier, M. Kasper, and J. C. Christou. Suppressing stellar residual light on extremely large telescopes by aperture modulation. In proceedings of Adaptive Optics for Extremely Large Telescopes, 2009.

6. Szymon Gladysz, Julian C. Christou, and Michael Refern. Characterization of the lick adaptive optics point spread function. Proc. SPIE, 6272, 2006.

7. R. A. Gonsalves. PHASE RETRIEVAL AND DIVERSITY IN ADAPTIVE OPTICS. Optical Engineering, 21(5), 1982

8. Jolissaint, Véran, and Conan. Analytical modeling of adaptive optics: foundations of the phase spatial power spectrum approach. JOSA, 23(2), February 2006. 